\title{
Robust Stability Analysis of Fractional-Order Hopfield Neural Networks with Parameter Uncertainties
}

\author{
Shuo Zhang, Yongguang Yu, and Wei Hu \\ Department of Mathematics, Beijing Jiaotong University, Beijing 100044, China \\ Correspondence should be addressed to Yongguang Yu; ygyu@bjtu.edu.cn
}

Received 14 February 2014; Accepted 4 March 2014; Published 6 April 2014

Academic Editor: Abdon Atangana

Copyright (C) 2014 Shuo Zhang et al. This is an open access article distributed under the Creative Commons Attribution License, which permits unrestricted use, distribution, and reproduction in any medium, provided the original work is properly cited.

The issue of robust stability for fractional-order Hopfield neural networks with parameter uncertainties is investigated in this paper. For such neural system, its existence, uniqueness, and global Mittag-Leffler stability of the equilibrium point are analyzed by employing suitable Lyapunov functionals. Based on the fractional-order Lyapunov direct method, the sufficient conditions are proposed for the robust stability of the studied networks. Moreover, robust synchronization and quasi-synchronization between the class of neural networks are discussed. Furthermore, some numerical examples are given to show the effectiveness of our obtained theoretical results.

\section{Introduction}

During the last decades, neural networks have received increasing attention in various fields, such as image and signal processing, associative memory, pattern recognition, optimization, control, and modelling. The Hopfield neural model is one of the most popular neural models in the previous literature. Thus, for integer-order Hopfield neural networks, it is important to study their dynamical properties, in particular, the stability analysis of integer-order Hopfield neural networks which is a prime issue for the practical design and application of neural networks. In recent years, some sufficient conditions for global asymptotic and exponential stability of integer-order Hopfield neural networks have been proposed [1-4]. However, the above researches did not consider the influence of parameter uncertainties, which are unavoidable due to measure errors, the parameter fluctuation, external disturbance, and so forth. For example, in electronic implementation of neural networks, some essential parameters such as release rate of neurons, connection weights between the neurons, and the transmission delays might be subject to some deviations owing to the tolerances of electronic components. Therefore, it is necessary to ensure that system be stable with respect to these uncertainties in the design and applications of neural networks. In other words, the designed neural network must be robust against such uncertainties. Nowadays, many researchers have studied the existence, uniqueness, and globally robust asymptotic stability of the equilibrium point in integer-order neural networks with parameter uncertainties and given some robust stability conditions [5-12]. To establish the robust stability criteria, Lyapunov functionals and linear matrix inequality (LMI) are two effective tools utilized in [5-8] and [9-12], respectively.

It is well known that chaos synchronization has attracted considerable attention due to its great potential applications in secure communication, biological science, engineering, and so on. It is worth noting that integer-order neural networks can exhibit some complicated dynamics and even chaotic behavior if the parameters are appropriately chosen $[13,14]$. Thus, the synchronization for integer-order chaotic neural networks has been a much-discussed topic and the robust synchronization issue for integer-order chaotic neural networks with parameter uncertainties is also very hot $[15,16]$. Similar to the robust stability of integer-order neural networks, Lyapunov functionals and LMI are two commonly used tools to analyze the robust synchronization issue between two integer-order chaotic neural networks with parameter uncertainties. However, when the parameter uncertainties between two integer-order chaotic neural networks are different, parameter mismatches would appear unavoidably. In this case, the zero equilibrium point of the error system may not exist, and it is impossible to achieve 
the complete synchronization. But many researchers found that the synchronization error converges to a small region around zero which is called quasi-synchronization or weak synchronization and gained much research attention [17-19].

All the above researches investigated only integer-order neural networks. In fact, as a generalization of integerorder differentiation and integration to arbitrary noninteger order, fractional-order calculus exists in both theoretical and applied aspects of numerous branches of science and engineering. Especially, using fractional derivative, the description of some systems is more accurate than integerorder systems, such as viscoelastic materials, electrochemical processes, long lines, dielectric polarisations, coloured noise, and cardiac behaviour [20-23]. Therefore, neural networks have received growing interest of scholars to build their fractional-order Hopfield models and study the characteristics of them, which show higher nonlinearity and more degrees of freedom than integer-order models [24, 25]. For the stability analysis of fractional-order neural networks, the linear stability theory of fractional-order system is the common method [26]. Moreover, in [26], Kaslik and Sivasundaram discussed the bifurcations and chaos of the nonlinear fractional-order Hopfield neural networks, which is very meaningful for synchronization between fractionalorder Hopfield neural networks. Sabatier et al. [27] proposed LMI stability conditions for fractional-order systems, which provides an approach to solving robust stability and synchronization issue of fractional-order systems with parameter uncertainties [28-31]. Liao et al. [28] studied robust stability for fractional-order linear time-invariant (FO-LTI) interval systems with parameter uncertainties. Wong et al. [31] investigated robust synchronization of fractional-order complex dynamical networks with parameter uncertainties. What a pity, no research discussed robust stability and synchronization for fractional-order Hopfield neural networks with parameter uncertainties. Besides, the above results are all in the form of LMI, and no scholar has ever employed the Lyapunov functional method which needs less calculation than LMI. Therefore, we try to deal with these problems and employ the Lyapunov functional to analyze the robust stability and synchronization for fractional-order Hopfield neural networks with parameter uncertainties.

It must be pointed out that Lyapunov stability theorem of integer-order systems cannot be used in fractional-order systems, until the fractional-order Lyapunov direct method was presented in [32]. The (generalized) Mittag-Leffler stability of fractional-order systems can be proved if the conditions of the fractional-order Lyapunov direct method are satisfied. So based on the fractional-order Lyapunov direct method, robust stability and synchronization for fractional-order systems can be analyzed by employing suitable Lyapunov functionals. Though for a multidimensional nonlinear fractionalorder system, a suitable Lyapunov functional is difficultly designed with satisfying the conditions of the fractionalorder Lyapunov direct method; one will be chosen and its suitability will be proved in this paper.

Motivated by the above discussion, robust stability and synchronization for fractional-order Hopfield neural networks with parameter uncertainties are studied. First, the globally robust stability of such neural system is analyzed via a Lyapunov functional, which has less calculation than LMI in early researches [28-31]. Besides, the Lyapunov functional is also employed to realize robust synchronization between two such neural systems with same parameter uncertainties. Moreover, the quasi-synchronization for this class of neural networks with different parameter uncertainties is investigated by a special Lyapunov functional, which is much simpler than the Laplace transform method used in [33]. In addition, numerical simulations are proposed to show their good agreement with the theoretical results.

The rest of the paper is organized as follows. In Section 2, some preliminaries are given, including Caputo fractionalorder derivative, Mittag-Leffler function, and Mittag-Leffler stability of fractional-order systems. Then, robust stability for fractional-order Hopfield neural networks with parameter uncertainties is proposed in Section 3. Robust synchronization and quasi-synchronization for fractional-order Hopfield neural networks with parameter uncertainties are presented in Section 4. Some examples in Section 5 are gained to verify the theoretical results. Finally, the paper is concluded in Section 6.

\section{Preliminaries}

2.1. Caputo Fractional-Order Derivative. As an important role in nonlinear science, fractional-order calculus has three common definitions, such as Grunwald-Letnikov, RiemannLiouville, and Caputo definitions [34]. Especially, Caputo fractional-order derivative owns same initial conditions with integer-order derivatives, which is well-understood in physical situations and more applicable to real world problems. Thus, we use the Caputo fractional-order derivative in this paper.

Definition 1 (Caputo fractional-order derivative). The Caputo fractional-order derivative of order $\alpha>0$ for a function $f(t) \in C^{n+1}\left(\left[t_{0},+\infty\right), R\right)$ is defined as

$$
{ }_{t_{0}} D_{t}^{\alpha} f(t)=\frac{1}{\Gamma(n-\alpha)} \int_{t_{0}}^{t} \frac{f^{(n)}(\tau)}{(t-\tau)^{\alpha+1-n}} d \tau,
$$

where $\Gamma(\cdot)$ denotes the Gamma function and $n$ is a positive integer such that $n-1<\alpha \leq n$.

The Laplace transform of the Caputo fractional-order derivative is

$$
\begin{array}{r}
\mathscr{L}\left\{{ }_{t_{0}} D_{t}^{\alpha} f(t) ; s\right\}=s^{\alpha} F(s)-\sum_{k=0}^{n-1} s^{\alpha-k-1} f^{(k)}\left(t_{0}\right), \\
n-1<\alpha \leq n,
\end{array}
$$

where $\mathscr{L}\{\cdot\}$ denotes the Laplace transform and $s$ is the variable in Laplace domain.

Property 1 . When $C$ is any constant, ${ }_{t_{0}} D_{t}^{\alpha} C=0$ holds.

Property 2. For constants $\mu$ and $\nu$, the linearity of Caputo fractional-order derivative is described by

$$
{ }_{t_{0}} D_{t}^{\alpha}(\mu f(t)+v g(t))=\mu_{t_{0}} D_{t}^{\alpha} f(t)+v_{t_{0}} D_{t}^{\alpha} g(t) \text {. }
$$


2.2. Mittag-Leffler Function. Mittag-Leffler function is frequently used in the solutions of fractional-order differential equations, which is similar to exponential function used in the solutions of integer-order differential equations.

Definition 2 (see [34]). The Mittag-Leffler function with two parameters is defined as

$$
E_{\alpha, \beta}(z)=\sum_{k=0}^{\infty} \frac{z^{k}}{\Gamma(\alpha k+\beta)}
$$

where $\alpha>0, \beta>0$, and $z \in C$. When $\beta=1$, its one-parameter form is shown as

$$
E_{\alpha}(z)=\sum_{k=0}^{\infty} \frac{z^{k}}{\Gamma(\alpha k+1)}=E_{\alpha, 1}(z) .
$$

In particular, $E_{1,1}(z)=e^{z}$. The Laplace transform of MittagLeffler function with two parameters is

$$
\mathscr{L}\left\{t^{\beta-1} E_{\alpha, \beta}\left(-\lambda t^{\alpha}\right)\right\}=\frac{s^{\alpha-\beta}}{s^{\alpha}+\lambda}, \quad\left(\operatorname{Re}(s)>|\lambda|^{1 / \alpha}\right),
$$

where $t \geq 0, s$ is the variable in Laplace domain, and $\operatorname{Re}(s)$ is the real part of $s, \lambda \in R$.

2.3. Mittag-Leffler Stability of Fractional-Order System. Consider the following $\mathrm{n}$-dimensional Caputo fractional-order system:

$$
\begin{gathered}
t_{0} D_{t}^{\alpha} x(t)=f(t, x(t)), \\
x\left(t_{0}\right)=x_{t_{0}},
\end{gathered}
$$

where $\alpha \in(0,1), x=\left(x_{1}, x_{2}, \ldots, x_{n}\right)^{T} \in R^{n}, t_{0} \geq 0$ is the initial time, and $f:[0,+\infty) \times R^{n} \rightarrow R^{n}$ is piecewise continuous on $t$ and satisfies locally Lipschitz condition on $x$.

Definition 3. The constant $\bar{x}$ is an equilibrium point of Caputo fractional-order dynamic system (7) if and only if $f(t, \bar{x})=0$.

Remark 4. Based on Properties 1 and 2, any equilibrium point can be translated to the origin via a change of variables. When the equilibrium point in (7) is $\bar{x} \neq 0$, system (7) with the change of variable $y(t)=x(t)-\bar{x}$ can be rewritten as

$$
\begin{aligned}
{ }_{t_{0}} D_{t}^{\alpha} y(t) & ={ }_{t_{0}} D_{t}^{\alpha}(x(t)-\bar{x})=f(t, x(t)) \\
& =f(t, y(t)+\bar{x})=g(t, y),
\end{aligned}
$$

where $g(t, 0)=0$ and the new system has equilibrium point at the origin for new variable $y$. Thus, without loss of generality, all definitions and theorems are gained for the cases when the equilibrium point is the origin; that is, $\bar{x}=0$.

Lemma 5 (Existence and uniqueness Theorem in [35]). There exists a unique solution of system (7), if system (7) has equilibrium point at the origin and $f(t, x)$ satisfies locally Lipschitz condition on $x$.
Definition 6 (Mittag-Leffler stability [32]). If $\bar{x}=0$ is an equilibrium point of system (7), the solution of (7) is said to be Mittag-Leffler stable if

$$
\|x(t)\| \leq\left[m\left(x_{t_{0}}\right) E_{\alpha}\left(-\lambda\left(t-t_{0}\right)^{\alpha}\right)\right]^{b},
$$

where $\lambda>0, b>0, m(0)=0,\|\cdot\|$ denotes an arbitrary norm, and $m(x) \geq 0$ satisfies locally Lipschitz condition on $x \in R^{n}$ with Lipschitz constant $m_{0}$.

Definition 7 (generalized Mittag-Leffler stability [32]). If $\bar{x}=$ 0 is an equilibrium point of system (7), the solution of (7) is said to be generalized Mittag-Leffler stable if

$$
\|x(t)\| \leq\left[m\left(x_{t_{0}}\right)\left(t-t_{0}\right)^{-\gamma} E_{\alpha, 1-\gamma}\left(-\lambda\left(t-t_{0}\right)^{\alpha}\right)\right]^{b},
$$

where $-\alpha<\gamma \leq 1-\alpha, \lambda>0, b>0, m(0)=0, m(x) \geq 0$, and $m(x)$ satisfies locally Lipschitz condition on $x \in R^{n}$ with Lipschitz constant $m_{0}$.

Remark 8. Both Mittag-Leffler stability and generalized Mittag-Leffler stability imply asymptotic stability; that is, $\|x(t)\| \rightarrow 0$ with $t \rightarrow+\infty$.

Remark 9. When the equilibrium point $\bar{x} \neq 0$, the solution of system (7) is said to be Mittag-Leffler stable (or generalized Mittag-Leffler stable) if

$$
\begin{gathered}
\|x(t)-\bar{x}\| \leq\left[m\left(x_{t_{0}}-\bar{x}\right) E_{\alpha}\left(-\lambda\left(t-t_{0}\right)^{\alpha}\right)\right]^{b}, \\
(\|x(t)-\bar{x}\| \\
\left.\quad \leq\left[m\left(x_{t_{0}}-\bar{x}\right)\left(t-t_{0}\right)^{-\gamma} E_{\alpha, 1-\gamma}\left(-\lambda\left(t-t_{0}\right)^{\alpha}\right)\right]^{b}\right),
\end{gathered}
$$

and $x(t) \rightarrow \bar{x}$ satisfies $t \rightarrow+\infty$.

In order to analyze Mittag-Leffler stability of system (7), the fractional-order Lyapunov direct method is introduced as follows.

Lemma 10 (fractional-order Lyapunov direct method [32]). For $t_{0}=0$, the fractional-order system (7) is Mittag-Leffler stable at the equilibrium point $\bar{x}=0$ if there exists a continuously differentiable function $V(t, x(t))$ that satisfies

$$
\begin{gathered}
\alpha_{1}\|x\|^{a} \leq V(t, x(t)) \leq \alpha_{2}\|x\|^{a b}, \\
{ }_{0} D_{t}^{\beta} V(t, x(t)) \leq-\alpha_{3}\|x\|^{a b},
\end{gathered}
$$

where $V(t, x(t)):[0, \infty) \times D \rightarrow R$ satisfies locally Lipschitz condition on $x ; D \subset R^{n}$ is a domain containing the origin; $t \geq 0$, $\beta \in(0,1), \alpha_{1}, \alpha_{2}, \alpha_{3}, a$, and $b$ are arbitrary positive constants. If the assumptions hold globally on $R^{n}$, then $\bar{x}=0$ is globally Mittag-Leffler stable.

Remark 11. According to the proof of fractional-order Lyapunov direct method in [32], the condition in Lemma 10 can be weakened. If the inequality in (13) holds almost everywhere, the result of Lemma 10 also holds. 
Lemma 12. If $h(t) \in C^{1}([0,+\infty), R)$ denotes a continuously differentiable function, the following inequality holds almost everywhere:

$$
\begin{array}{r}
{ }_{0} D_{t}^{\alpha}|h(t)| \leq \operatorname{sgn}(h(t)){ }_{0} D_{t}^{\alpha} h(t), \\
0<\alpha<1 .
\end{array}
$$

Proof. Without loss of generality, the trajectory of $|h(t)|$ can be simply described as the solid line in Figure 1. The $|h(t)|$ is differentiable except at the points $P_{3}$ and $P_{5}$, which are the solutions of $h(t)=0$. The trajectory is divided into three parts by the points $P_{3}$ and $P_{5}$, and $P_{2}$ and $P_{4}$ are the extreme points. Point $P_{1}$ is the initial date $|h(0)|$. The dash line is the trajectory of $-|h(t)|$ which may be the one of $h(t)$ in any part. $\left(x_{P}, y_{P}\right)$ denotes the coordinate of point $P$ in Figure 1.

In Part $A$, if $\operatorname{sgn}(h(t)) h(\tau)=|h(\tau)|$, we obtain $\int_{0}^{x_{P_{3}}}\left(|h(\tau)|^{\prime} /(t-\tau)^{\alpha}\right) d \tau=\int_{0}^{x_{P_{3}}}\left((\operatorname{sgn}(h(t)) h(\tau))^{\prime} /(t-\tau)^{\alpha}\right) d \tau$; if $\operatorname{sgn}(h(t)) h(\tau)=-|h(\tau)|$, then

$$
\begin{aligned}
\int_{0}^{x_{P_{3}}} & \frac{|h(\tau)|^{\prime}}{(t-\tau)^{\alpha}} d \tau \\
= & \int_{0}^{x_{P_{2}}} \frac{|h(\tau)|^{\prime}}{(t-\tau)^{\alpha}} d \tau+\int_{x_{P_{2}}}^{x_{P_{3}}} \frac{|h(\tau)|^{\prime}}{(t-\tau)^{\alpha}} d \tau \\
< & \frac{1}{\left(t-x_{P_{2}}\right)^{\alpha}} \int_{0}^{x_{P_{2}}}|h(\tau)|^{\prime} d \tau+\frac{1}{\left(t-x_{P_{2}}\right)^{\alpha}} \\
& \times \int_{x_{P_{2}}}^{x_{P_{3}}}|h(\tau)|^{\prime} d \tau \\
= & \frac{-y_{P_{1}}}{\left(t-x_{P_{2}}\right)^{\alpha}} \leq 0 .
\end{aligned}
$$

According to $\operatorname{sgn}(h(t)) h(\tau)=-|h(\tau)|$, we get

$$
\begin{aligned}
& \int_{0}^{x_{P_{3}}} \frac{(\operatorname{sgn}(h(t)) h(\tau))^{\prime}}{(t-\tau)^{\alpha}} d \tau \\
& \quad=-\int_{0}^{x_{P_{3}}} \frac{|h(\tau)|^{\prime}}{(t-\tau)^{\alpha}} d \tau>0>\int_{0}^{x_{P_{3}}} \frac{|h(\tau)|^{\prime}}{(t-\tau)^{\alpha}} d \tau .
\end{aligned}
$$

Using the same method, Parts $B$ and $C$ have similar result. So we obtain

$$
\begin{aligned}
{ }_{0} D_{t}^{\alpha}|h(t)| & =\frac{1}{\Gamma(1-\alpha)} \int_{0}^{t} \frac{|h(\tau)|^{\prime}}{(t-\tau)^{\alpha}} d \tau \\
& \leq \frac{1}{\Gamma(1-\alpha)} \int_{0}^{t} \frac{(\operatorname{sgn}(h(t)) h(\tau))^{\prime}}{(t-\tau)^{\alpha}} d \tau \\
& =\operatorname{sgn}(h(t)){ }_{0} D_{t}^{\alpha} h(t)
\end{aligned}
$$

except at the zero points $\left(P_{3}\right.$ and $\left.P_{5}\right)$.

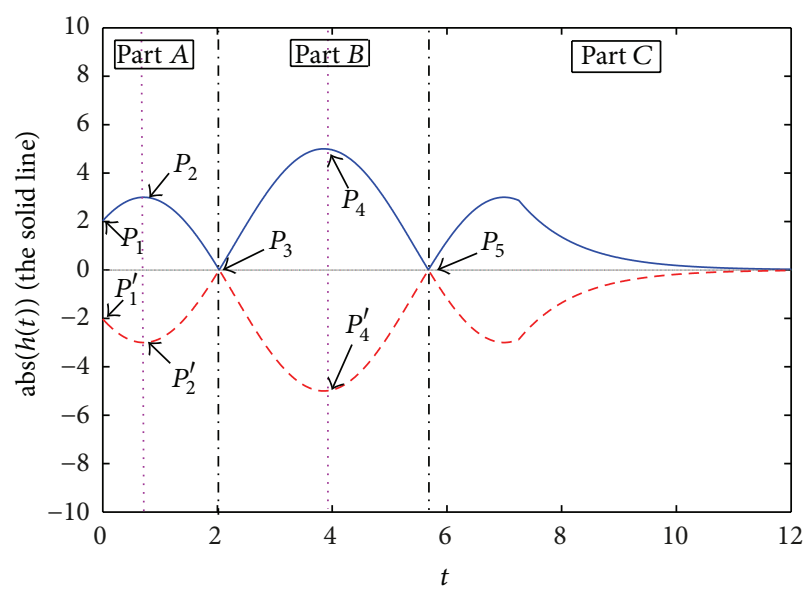

FiguRE 1: A simple example of $|h(t)|$ s trajectory.

\section{Robust Stability for Fractional-Order Hopfield Neural Networks with Parameter Uncertainties}

Consider the following $n$-dimensional Caputo fractionalorder Hopfield neural networks:

$$
{ }_{0} D_{t}^{\alpha} x(t)=-(A+\Delta A(t)) x(t)+(B+\Delta B(t)) f(x(t))+w,
$$

where $\alpha \in(0,1), x(t)=\left(x_{1}(t), x_{2}(t), \ldots, x_{n}(t)\right)^{T} \in R^{n}$, $f(x(t))=\left(f_{1}\left(x_{1}\right), f_{2}\left(x_{2}\right), \ldots, f_{n}\left(x_{n}\right)\right)^{T} \in R^{n}, A=\operatorname{diag}\left\{a_{1}, a_{2}\right.$, $\left.\ldots, a_{n}\right\}$, and $B=\left(b_{i j}\right)_{n \times n}$. For $i, j=1,2, \ldots, n, x_{i}(t)$ is the state of the $i$ th unit at time $t, f_{i}\left(x_{i}\right)$ denotes the activation function of the $i$ th neuron, $a_{i}>0$ denotes the charging rate for the $i$ th neuron, and $b_{i j}$ is the constant connection weight of the $j$ th neuron on the $i$ th neuron. $\Delta A(t)=$ $\operatorname{diag}\left\{\Delta a_{1}(t), \Delta a_{2}(t), \ldots, \Delta a_{n}(t)\right\}$ and $\Delta B(t)=\left(\Delta b(t)_{i j}\right)_{n \times n}$ are matrices with time-varying parametric uncertainties. $w=$ $\left(w_{1}, w_{2}, \ldots, w_{n}\right)^{T}$ is the constant external input vector.

In the rest of this paper, $\|Q\|$ denotes the 1-norm of corresponding vector $Q$ or matrix $Q$. When $Q=$ $\left(Q_{1}, Q_{2}, \ldots, Q_{m}\right)^{T} \in R^{m}$ is a vector, $\|Q\|=\left|Q_{1}\right|+\left|Q_{2}\right|+$ $\cdots+\left|Q_{m}\right|$. If $Q \in R^{m \times m}$ is a matrix, $\|Q\|=\sup \{\|Q x\|:$ $\left.\forall x \in R^{m},\|x\| \leq 1\right\}$. An $m$-dimensional vector $Q=$ $\left(Q_{1}, Q_{2}, \ldots, Q_{m}\right)^{T}>0(\geq 0)$ represents any $Q_{i}>0(\geq 0)$ for $i=1,2, \ldots, m$. If $P, Q \in R^{m}, P>(\geq) Q$ means $P-Q>(\geq) 0$.

Based on the above formulations, the concept of robust stability for fractional-order Hopfield neural networks with parameter uncertainties is introduced by the following definition.

Definition 13. The fractional-order Hopfield neural networks (18) are globally asymptotically robust stable if the unique equilibrium point $\bar{x}$ of the neural system is globally asymptotically stable under the time-varying parameter uncertainties.

In order to obtain globally asymptotically robust stability of system (18), three assumptions are given as follows.

$\left(A_{1}\right)$ Matrices with time-varying parametric uncertainties $\Delta A(t)$ and $\Delta B(t)$ are bounded: there exist constants 
$M_{A}, M_{B}>0$ such that $\|\Delta A(t)\| \leq M_{A}$ and $\|\Delta B(t)\| \leq$ $M_{B}$.

$\left(A_{2}\right)$ Activation functions $f_{i}$ are continuous and satisfy Lipschitz condition on $R$ with Lipschitz constant $l_{i}>$ 0 ; that is,

$$
\left|f_{i}(x)-f_{i}(y)\right| \leq l_{i}|x-y|
$$

for all $x, y \in R$ and $i=1,2, \ldots, n$, which ensures the existence and uniqueness of the solutions of system (18) based on Lemma 5.

$\left(A_{3}\right)$ There exist positive constants $\lambda$ and $\beta_{i}(i=1,2, \ldots, n)$ such that

$$
\left(A-L|B|^{T}\right)\left(\begin{array}{c}
\beta_{1} \\
\beta_{2} \\
\vdots \\
\beta_{n}
\end{array}\right) \geq\left(M_{A}\|\beta\|+M_{B}\|L\|\|\beta\|+\lambda\right)\left(\begin{array}{c}
1 \\
1 \\
\vdots \\
1
\end{array}\right),
$$

where $|B|=\left(\left|b_{i j}\right|\right)_{n \times n}, L=\operatorname{diag}\left\{l_{1}, l_{2}, \ldots, l_{n}\right\}$, and $\beta=$ $\operatorname{diag}\left\{\beta_{1}, \beta_{2}, \ldots, \beta_{n}\right\}$.

Theorem 14. Under assumptions $\left(A_{1}\right)-\left(A_{3}\right)$, system (18) is globally asymptotically robust stable.

Proof. Firstly, we need to prove the existence and uniqueness of equilibrium point of system (18). Define a mapping $H(\mu)=$ $\left(H_{1}(\mu), H_{2}(\mu), \ldots, H_{n}(\mu)\right)^{T}$, where $\mu=\left(\mu_{1}, \mu_{2}, \ldots, \mu_{n}\right)^{T} \in R^{n}$ and

$$
\begin{array}{r}
H_{i}(\mu)=\frac{\Delta a_{i}(t) \mu_{i}}{a_{i}}+\beta_{i} \sum_{j=1}^{n}\left(b_{i j}+\Delta b_{i j}(t)\right) f_{j}\left(\frac{\mu_{j}}{a_{j} \beta_{j}}\right)+\beta_{i} w_{i}, \\
(i=1,2, \ldots, n) .
\end{array}
$$

According to $\left(A_{2}\right)$, for any two vectors $\mu, v \in R^{n}$, we obtain

$$
\begin{aligned}
\left|H_{i}(\mu)-H_{i}(\nu)\right|= & \frac{\left|\Delta a_{i}(t)\right|}{a_{i}}\left|\mu_{i}-v_{i}\right| \\
& +\beta_{i} \mid \sum_{j=1}^{n}\left(b_{i j}+\Delta b_{i j}(t)\right) \\
& \times\left[f_{j}\left(\frac{\mu_{j}}{a_{j} \beta_{j}}\right)-f_{j}\left(\frac{v_{j}}{a_{j} \beta_{j}}\right)\right] \mid \\
\leq & \frac{\left|\Delta a_{i}(t)\right|}{a_{i}}\left|\mu_{i}-v_{i}\right| \\
& +\beta_{i} \sum_{j=1}^{n} \frac{\left(\left|b_{i j}\right|+\left|\Delta b_{i j}(t)\right|\right) l_{j}}{a_{j} \beta_{j}}\left|\mu_{j}-v_{j}\right| .
\end{aligned}
$$

Then, we have

$$
\begin{aligned}
\| H & (\mu)-H(\nu) \| \\
= & \sum_{i=1}^{n}\left|H_{i}(\mu)-H_{i}(v)\right| \\
\leq & \sum_{i=1}^{n} \frac{\left|\Delta a_{i}(t)\right|}{a_{i}}\left|\mu_{i}-v_{i}\right| \\
& +\sum_{i=1}^{n} \sum_{j=1}^{n} \frac{\left(\left|b_{i j}\right|+\left|\Delta b_{i j}(t)\right|\right) \beta_{i} l_{j}}{a_{j} \beta_{j}}\left|\mu_{j}-v_{j}\right| \\
= & \sum_{i=1}^{n} \frac{\left|\Delta a_{i}(t)\right|}{a_{i}}\left|\mu_{i}-v_{i}\right| \\
& +\sum_{i=1}^{n} \sum_{j=1}^{n} \frac{\left(\left|b_{j i}\right|+\left|\Delta b_{j i}(t)\right|\right) \beta_{j} l_{i}}{a_{i} \beta_{i}}\left|\mu_{i}-v_{i}\right| \\
= & \sum_{i=1}^{n} \frac{\left|\Delta a_{i}(t)\right| \beta_{i}+\sum_{j=1}^{n}\left(\left|b_{j i}\right|+\left|\Delta b_{j i}(t)\right|\right) \beta_{j} l_{i}}{a_{i} \beta_{i}} \\
& \quad \times\left|\mu_{i}-v_{i}\right| .
\end{aligned}
$$

From $\left(A_{3}\right)$, for any $i=1,2, \ldots, n$, we get

$$
\begin{aligned}
a_{i} \beta_{i}- & \left(\sum_{j=1}^{n}\left|b_{j i}\right| \beta_{j} l_{i}+\left|\Delta a_{i}(t)\right| \beta_{i}+\sum_{j=1}^{n}\left|\Delta b_{j i}(t)\right| \beta_{j} l_{i}\right) \\
\geq & a_{i} \beta_{i}-\sum_{j=1}^{n}\left|b_{j i}\right| \beta_{j} l_{i} \\
& -\|\Delta A(t) \beta\|-\|\beta \Delta B(t) L\| \\
\geq & a_{i} \beta_{i}-\sum_{j=1}^{n}\left|b_{j i}\right| \beta_{j} l_{i}-M_{A}\|\beta\| \\
& -M_{B}\|L\|\|\beta\| \geq \lambda>0 .
\end{aligned}
$$

So from inequalities (23) and (24), we gain

$$
\|H(\mu)-H(\nu)\|<\sum_{i=1}^{n}\left|\mu_{i}-v_{i}\right|=\|\mu-v\|
$$

which means the mapping $H: R^{n} \rightarrow R^{n}$ is a contraction mapping on $R^{n}$. Thus, there exists a unique fixed point $\bar{\mu} \in R^{n}$ such that $H(\bar{\mu})=\bar{\mu}$; that is,

$$
\begin{array}{r}
\bar{\mu}_{i}=\frac{\Delta a_{i}(t) \bar{\mu}_{i}}{a_{i}}+\beta_{i} \sum_{j=1}^{n}\left(b_{i j}+\Delta b_{i j}(t)\right) f_{j}\left(\frac{\bar{\mu}_{j}}{a_{j} \beta_{j}}\right)+\beta_{i} w_{i}, \\
(i=1,2, \ldots, n) .
\end{array}
$$


Denote $\bar{x}_{i}=\bar{\mu}_{i} / a_{i} \beta_{i}$ for $i=1,2, \ldots, n$, then

$$
\begin{array}{r}
-\left(a_{i}+\Delta a_{i}(t)\right) \bar{x}_{i}+\sum_{j=1}^{n}\left(b_{i j}+\Delta b_{i j}(t)\right) f_{j}\left(\bar{x}_{j}\right)+w_{i}=0 \\
(i=1,2, \ldots, n) .
\end{array}
$$

Thus, we have

$$
-(A+\Delta A(t)) \bar{x}+(B+\Delta B(t)) f(\bar{x})+w=0
$$

where $\bar{x}=\left(\bar{x}_{1}, \bar{x}_{2}, \ldots, \bar{x}_{n}\right)^{T}$ is the unique equilibrium of system (18).

Secondly, let us prove that system (18) is globally asymptotically robust stable. Let $x(t)$ and $y(t)$ be any two solutions of system (37) with different initial values. We can obtain the error system with error $e(t)=\left(e_{1}(t), e_{2}(t), \ldots, e_{n}(t)\right)^{T}=$ $y(t)-x(t)$ :

$$
\begin{aligned}
{ }_{0} D_{t}^{\alpha} e(t)= & -(A+\Delta A(t)) e(t) \\
& +(B+\Delta B(t))(f(y(t))-f(x(t))) .
\end{aligned}
$$

Construct a Lyapunov functional as

$$
V(t, e(t))=\sum_{i=1}^{n} \beta_{i}\left|e_{i}(t)\right| .
$$

It is obvious that the Lyapunov functional (30) satisfies the condition as the inequality in (12). Then, we are going to prove that the Lyapunov functional (30) also satisfies the condition as the inequality in (13) almost everywhere.

According to Definition $1, e_{i}(t) \in C^{1}([0,+\infty), R)$ is continuously differentiable. Due to Assumptions $\left(A_{1}\right)-\left(A_{3}\right)$, Lemma 12, and (24), the following inequality holds almost everywhere:

$$
\begin{aligned}
{ }_{0} D_{t}^{\alpha} V(t, e(t)) & \\
= & \sum_{i=1}^{n} \beta_{i_{0}} D_{t}^{\alpha}\left|e_{i}(t)\right| \leq \sum_{i=1}^{n} \beta_{i} \operatorname{sgn}\left(e_{i}(t)\right)_{0} D_{t}^{\alpha} e_{i}(t) \\
=\sum_{i=1}^{n} \beta_{i} \operatorname{sgn}\left(e_{i}(t)\right) & \\
\times & {\left[\begin{array}{l}
-\left(a_{i}+\Delta a_{i}(t)\right) e_{i}(t) \\
+\sum_{j=1}^{n}\left(b_{i j}+\Delta b_{i j}(t)\right) \\
\times\left(f_{j}\left(y_{j}(t)\right)-f_{j}\left(x_{j}(t)\right)\right)
\end{array}\right] }
\end{aligned}
$$

$$
\begin{aligned}
& \leq \sum_{i=1}^{n} \beta_{i}\left[-a_{i}\left|e_{i}(t)\right|+\left|\Delta a_{i}(t)\right|\left|e_{i}(t)\right|\right. \\
& \left.+\sum_{j=1}^{n} l_{j}\left(\left|b_{i j}\right|+\left|\Delta b_{i j}(t)\right|\right)\left|e_{i}(t)\right|\right] \\
& =\sum_{i=1}^{n} \beta_{i}\left(-a_{i}\left|e_{i}(t)\right|+\left|\Delta a_{i}(t)\right|\left|e_{i}(t)\right|\right) \\
& +\sum_{i=1}^{n} \sum_{j=1}^{n} \beta_{j} l_{i}\left(\left|b_{j i}\right|+\left|\Delta b_{j i}(t)\right|\right)\left|e_{i}(t)\right| \\
& =-\sum_{i=1}^{n}\left(a_{i} \beta_{i}-\sum_{j=1}^{n}\left|b_{j i}\right| \beta_{j} l_{i}\right. \\
& \left.-\left|\Delta a_{i}(t)\right| \beta_{i}-\sum_{j=1}^{n}\left|\Delta b_{j i}(t)\right| \beta_{j} l_{i}\right)\left|e_{i}(t)\right| \\
& \leq-\lambda \sum_{i=1}^{n}\left|e_{i}(t)\right|=-\lambda\|e(t)\| .
\end{aligned}
$$

Thus, based on Lemma 10, the error system (29) is globally Mittag-Leffler stable; that is,

$$
\|e(t)\| \leq V(0, e(0)) E_{\alpha}\left(-\lambda t^{\alpha}\right) .
$$

Due to the fact that $\bar{x}$ is the unique equilibrium of system (37), we have

$$
\|x(t)-\bar{x}\| \leq V(0, x(0)-\bar{x}) E_{\alpha}\left(-\lambda t^{\alpha}\right),
$$

for any solution of system (37).

Therefore, the unique equilibrium point $\bar{x}$ of system (18) is globally Mittag-Leffler stable and the fractional-order Hopfield neural networks (18) with parameter uncertainties are globally asymptotically robust stable.

Remark 15. In Theorem 14, the robust stability of system (18) is deduced from its Mittag-Leffler stability. Based on the fractional-order Lyapunov direct method, the global Mittag-Leffler stability of system (18) can be guaranteed by employing the Lyapunov functional techniques. This method which is very convenient to implement in practice can be applied to almost all neural networks with the uniform Lipschitz activation functions. Moreover, it should be noted that the convergence rate is affected by the parameter $\lambda$ in MittagLeffler stability. In Theorem 14, the unique equilibrium point is faster converged to with a larger $\lambda$.

\section{Synchronization for Fractional-Order Hopfield Neural Networks with Parameter Uncertainties}

In this section, synchronization for fractional-order Hopfield neural networks with parameter uncertainties is investigated. For same or different parameter uncertainties, robust 
synchronization and quasi-synchronization for fractionalorder Hopfield neural networks are studied by employing the chosen Lyapunov functionals.

4.1. Robust Synchronization for Fractional-Order Hopfield Neural Networks with Same Parameter Uncertainties. Consider two chaotic fractional-order Hopfield neural networks with same parameter uncertainties as

$$
{ }_{0} D_{t}^{\alpha} x(t)=-(A+\Delta A(t)) x(t)+(B+\Delta B(t)) f(x(t))+w
$$

$$
\begin{aligned}
{ }_{0} D_{t}^{\alpha} y(t)= & -(A+\Delta A(t)) y(t) \\
& +(B+\Delta B(t)) f(y(t))+w+u(t),
\end{aligned}
$$

where $x(t)=\left(x_{1}(t), x_{2}(t), \ldots, x_{n}(t)\right)^{T}$ and $y(t)=\left(y_{1}(t)\right.$, $\left.y_{2}(t), \ldots, y_{n}(t)\right)^{T}$ are the state vectors of drive system (34) and response system (35), respectively. $\alpha, A, \Delta A(t), B, \Delta B(t)$, $f$, and $w$ are defined the same as the ones in system (18). $u(t)=\left(u_{1}(t), u_{2}(t), \ldots, u_{n}(t)\right)^{T}$ is the control law and $u(t)=$ $-k(y(t)-x(t))$, where $k$ is a positive constant.

Definition 16. Systems (34) and (35) with parameter uncertainties are said to realize robust synchronization, if error vector $e(t)=y(t)-x(t)$ converges to zero; that is,

$$
\lim _{t \rightarrow+\infty}\|e(t)\|=\lim _{t \rightarrow+\infty}\|y(t)-x(t)\|=0 .
$$

Theorem 17. For systems (34) and (35), the robust synchronization can be realized, if $\left(A_{1}\right)$ and $\left(A_{2}\right)$ hold and there exist positive constants $\lambda$ and $\beta_{i}(i=1,2, \ldots, n)$ such that

$$
\begin{aligned}
& \left(k I+A-L|B|^{T}\right)\left(\begin{array}{c}
\beta_{1} \\
\beta_{2} \\
\vdots \\
\beta_{n}
\end{array}\right) \\
& \quad \geq\left(M_{A}\|\beta\|+M_{B}\|L\|\|\beta\|+\lambda\right)\left(\begin{array}{c}
1 \\
1 \\
\vdots \\
1
\end{array}\right),
\end{aligned}
$$

where $|B|=\left(\left|b_{i j}\right|\right)_{n \times n}, L=\operatorname{diag}\left\{l_{1}, l_{2}, \ldots, l_{n}\right\}$, and $\beta=$ $\operatorname{diag}\left\{\beta_{1}, \beta_{2}, \ldots, \beta_{n}\right\}$.

Proof. For systems (34) and (35), the error system can be described as

$$
\begin{aligned}
{ }_{0} D_{t}^{\alpha} e(t)= & -(k I+A+\Delta A(t)) e(t) \\
& +(B+\Delta B(t))(f(y(t))-f(x(t))) .
\end{aligned}
$$

Based on Theorem 14, the unique equilibrium point $\bar{e}=0$ of system (38) is globally Mittag-Leffler stable; that is,

$$
\lim _{t \rightarrow+\infty}\|e(t)\|=0 \text {. }
$$

Thus, the robust synchronization between systems (34) and (35) is realized.
4.2. Quasi-Synchronization for Fractional-Order Hopfield Neural Networks with Different Parameter Uncertainties. Consider two chaotic fractional-order Hopfield neural networks with different parameter uncertainties as

$$
\begin{aligned}
{ }_{0} D_{t}^{\alpha} x(t)=- & (A+\Delta A(t)) x(t)+(B+\Delta B(t)) f(x(t))+w \\
& (40) \\
{ }_{0} D_{t}^{\alpha} y(t)= & -(A+\Delta C(t)) y(t)+(B+\Delta D(t)) f(y(t)) \\
& +w+u(t),
\end{aligned}
$$

where $x(t)=\left(x_{1}(t), x_{2}(t), \ldots, x_{n}(t)\right)^{T}$ and $y(t)=$ $\left(y_{1}(t), y_{2}(t), \ldots, y_{n}(t)\right)^{T}$ are the state vectors of drive system (40) and response system (41), respectively. $\alpha, A, B$, $f$, and $w$ are defined the same as the ones in system (18). $\Delta A(t)=\operatorname{diag}\left\{\Delta a_{1}(t), \Delta a_{2}(t), \ldots, \Delta a_{n}(t)\right\}, \Delta B(t)=$ $\left(\Delta b(t)_{i j}\right)_{n \times n}, \Delta C(t)=\operatorname{diag}\left\{\Delta c_{1}(t), \Delta c_{2}(t), \ldots, \Delta c_{n}(t)\right\}$, and $\Delta D(t)=\left(\Delta d(t)_{i j}\right)_{n \times n}$ are different matrices with time-varying parametric uncertainties. $u(t)=\left(u_{1}(t), u_{2}(t), \ldots, u_{n}(t)\right)^{T}$ is the control law and $u(t)=-k e(t)=-k(y(t)-x(t))$, where $k$ is a positive constant.

For systems (40) and (41), the error system can be written as

$$
\begin{aligned}
{ }_{0} D_{t}^{\alpha} e(t)= & -(k I+A+\Delta C(t)) e(t) \\
& +(B+\Delta D(t))(f(y(t))-f(x(t))) \\
& +(\Delta A(t)-\Delta C(t)) x(t) \\
& +(\Delta D(t)-\Delta B(t)) f(x(t)) .
\end{aligned}
$$

Owing to the differences of parameter uncertainties between systems (40) and (41), $\bar{e}=0$ is not the equilibrium point of error system (42). So the robust synchronization between systems (40) and (41) cannot be realized under the given control law $u$. However, the quasi-synchronization between systems (40) and (41) can be investigated under some conditions.

Definition 18. The quasi-synchronization between systems (40) and (41) is realized with error bound $\varphi \geq 0$ if there exists a $T \geq t_{0}$ such that for all $t \geq T$ and initial values $e(0)$,

$$
\|e(t)\|=\|y(t)-x(t)\| \leq \varphi .
$$

Then, the following assumptions and Lemma 19 are introduced to realize the quasi-synchronization between systems (40) and (41).

$\left(A_{4}\right)$ The state vector of drive system (40) is bounded: there exist constants $\delta$ such that $\|x(t)\| \leq \delta$. And function vector $f$ is bounded; that is, $\|f(x(t))\| \leq \delta_{f}$, for any $x$ which satisfies $\|x(t)\| \leq \delta$.

$\left(A_{5}\right)$ Matrices with time-varying parametric uncertainties $\Delta A(t), \Delta B(t), \Delta C(t)$, and $\Delta D(t)$ are bounded: there exist constants $M_{A}, M_{B}, M_{C}, M_{D}>0$ such that $\|\Delta A(t)\| \leq M_{A},\|\Delta B(t)\| \leq M_{B},\|\Delta C(t)\| \leq M_{C}$, and $\|\Delta D(t)\| \leq M_{D}$. 
$\left(A_{6}\right)$ There exists positive constant $\lambda$ such that

$$
\left(k I+A-L|B|^{T}\right)\left(\begin{array}{c}
1 \\
1 \\
\vdots \\
1
\end{array}\right) \geq\left(M_{C}+M_{D}\|L\|+\lambda\right)\left(\begin{array}{c}
1 \\
1 \\
\vdots \\
1
\end{array}\right) \text {, }
$$

where $|B|=\left(\left|b_{i j}\right|\right)_{n \times n}$ and $L=\operatorname{diag}\left\{l_{1}, l_{2}, \ldots, l_{n}\right\}$.

Lemma 19 (fractional-order comparison principle [36]). If ${ }_{0} D_{t}^{\alpha} x(t) \leq{ }_{0} D_{t}^{\alpha} y(t)$ with $0<\alpha<1$ and $x(0)=y(0)$, then $x(t) \leq y(t)$.

Theorem 20. If $\left(A_{2}\right)$ and $\left(A_{4}\right)-\left(A_{6}\right)$ hold, the quasisynchronization between systems $(40)$ and (41) can be realized with error bound $\varphi=\theta / \lambda+\epsilon$, where $0<\epsilon \ll 1$ is an arbitrary small constant and $\theta=n\left[\left(M_{A}+M_{C}\right) \delta+\left(M_{B}+M_{D}\right) \delta_{f}\right]$.

Proof. For the error system (42), choose a Lyapunov functional as

$$
V(t, e(t))=\|e(t)\|=\sum_{i=1}^{n}\left|e_{i}(t)\right|
$$

According to $\left(A_{2}\right),\left(A_{4}\right),\left(A_{5}\right)$, and Lemma 12, the following inequality holds almost everywhere:

$$
\begin{aligned}
& { }_{0} D_{t}^{\alpha} V(t, e(t)) \\
& =\sum_{i=1}^{n}{ }_{0} D_{t}^{\alpha}\left|e_{i}(t)\right| \leq \sum_{i=1}^{n} \operatorname{sgn}\left(e_{i}(t)\right)_{0} D_{t}^{\alpha} e_{i}(t) \\
& =\sum_{i=1}^{n} \operatorname{sgn}\left(e_{i}(t)\right) \\
& \times\left[-\left(k+a_{i}+\Delta c_{i}(t)\right) e_{i}(t)\right. \\
& +\sum_{j=1}^{n}\left(b_{i j}+\Delta d_{i j}(t)\right) \\
& \times\left(f_{j}\left(y_{j}(t)\right)-f_{j}\left(x_{j}(t)\right)\right) \\
& +\left(\Delta a_{i}(t)-\Delta c_{i}(t)\right) x_{i}(t) \\
& \left.+\sum_{j=1}^{n}\left(\Delta d_{i j}(t)-\Delta b_{i j}(t)\right) f_{j}\left(x_{j}(t)\right)\right] \\
& \leq \sum_{i=1}^{n}\left[-\left(k+a_{i}\right)\left|e_{i}(t)\right|+\left|\Delta c_{i}(t)\right|\left|e_{i}(t)\right|\right. \\
& +\sum_{j=1}^{n} l_{j}\left(\left|b_{i j}\right|+\left|\Delta d_{i j}(t)\right|\right)\left|e_{j}(t)\right|
\end{aligned}
$$

$$
\begin{gathered}
+\left(\left|\Delta a_{i}(t)\right|+\left|\Delta c_{i}(t)\right|\right)\left|x_{i}(t)\right| \\
+\sum_{j=1}^{n}\left(\left|\Delta d_{i j}(t)\right|+\left|\Delta b_{i j}(t)\right|\right) \\
\left.\times\left|f_{j}\left(x_{j}(t)\right)\right|\right]
\end{gathered}
$$

$$
\begin{aligned}
=\sum_{i=1}^{n}[- & \left(k+a_{i}\right)\left|e_{i}(t)\right|+\left|\Delta c_{i}(t)\right|\left|e_{i}(t)\right| \\
& +\sum_{j=1}^{n} l_{i}\left(\left|b_{j i}\right|+\left|\Delta d_{j i}(t)\right|\right)\left|e_{i}(t)\right| \\
& +\left(\left|\Delta a_{i}(t)\right|+\left|\Delta c_{i}(t)\right|\right)\left|x_{i}(t)\right| \\
& \left.+\sum_{j=1}^{n}\left(\left|\Delta d_{j i}(t)\right|+\left|\Delta b_{j i}(t)\right|\right)\left|f_{i}\left(x_{i}(t)\right)\right|\right]
\end{aligned}
$$$$
\leq-\sum_{j=1}^{n}\left(k+a_{i}-\left|\Delta c_{i}(t)\right|\right.
$$$$
\left.-\sum_{j=1}^{n} l_{i}\left(\left|b_{j i}\right|+\left|\Delta d_{j i}(t)\right|\right)\right)\left|e_{i}(t)\right|
$$$$
+n\left[\left(M_{A}+M_{C}\right) \delta+\left(M_{B}+M_{D}\right) \delta_{f}\right] \text {. }
$$

From $(24)$ and $\left(A_{6}\right)$, we obtain

$$
{ }_{0} D_{t}^{\alpha} V(t, e(t)) \leq-\lambda \sum_{i=1}^{n}\left|e_{i}(t)\right|+\theta=-\lambda V(t, e(t))+\theta \text {. }
$$

Then, consider the following one-dimensional fractionalorder system:

$$
{ }_{0} D_{t}^{\alpha} z(t)=-\lambda z(t)+\theta .
$$

According to Theorem 14 , the unique equilibrium point $\bar{z}=$ $\theta / \lambda$ of system (48) is globally Mittag-Leffler stable; that is,

$$
\lim _{t \rightarrow+\infty}\|z(t)-\bar{z}\|=0
$$

Due to (47), (48), and Lemma 19, we gain $V(t, e(t)) \leq z(t)$ with $V(0, e(0))=z(0)$. Thus, there exists a $T \geq t_{0}$ such that for all $t \geq T$,

$$
\|e(t)\| \leq \lim _{t \rightarrow+\infty} z(t)+\epsilon=\frac{\theta}{\lambda}+\epsilon,
$$

and the quasi-synchronization between systems (40) and (41) can be realized with error bound $\theta / \lambda+\epsilon$.

Remark 21. If the control parameter $k$ gets larger, the error bound $\varphi=\theta / \lambda+\epsilon$ will become smaller. So the synchronization error bound can be made as small as we want by choosing suitable control parameters. It is very important to chaos synchronization and nonlinear systems control. 


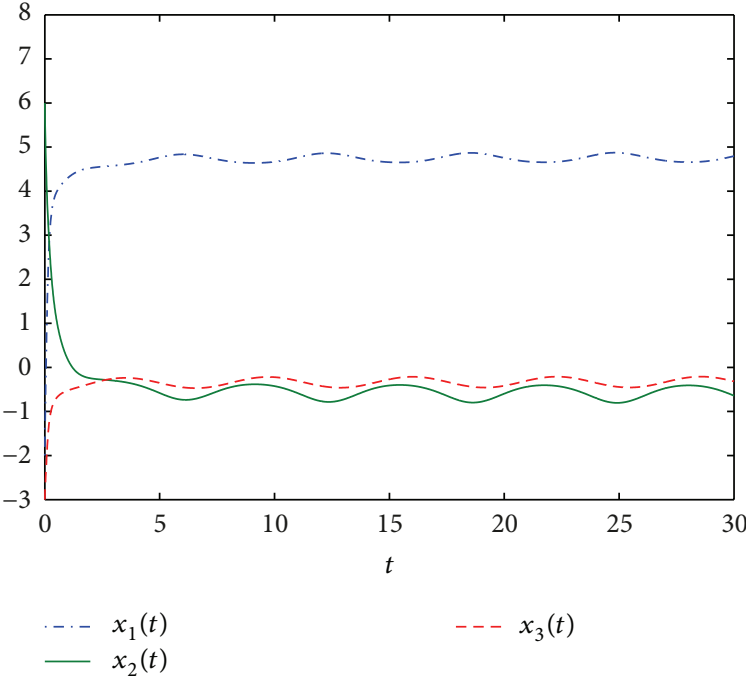

(a)

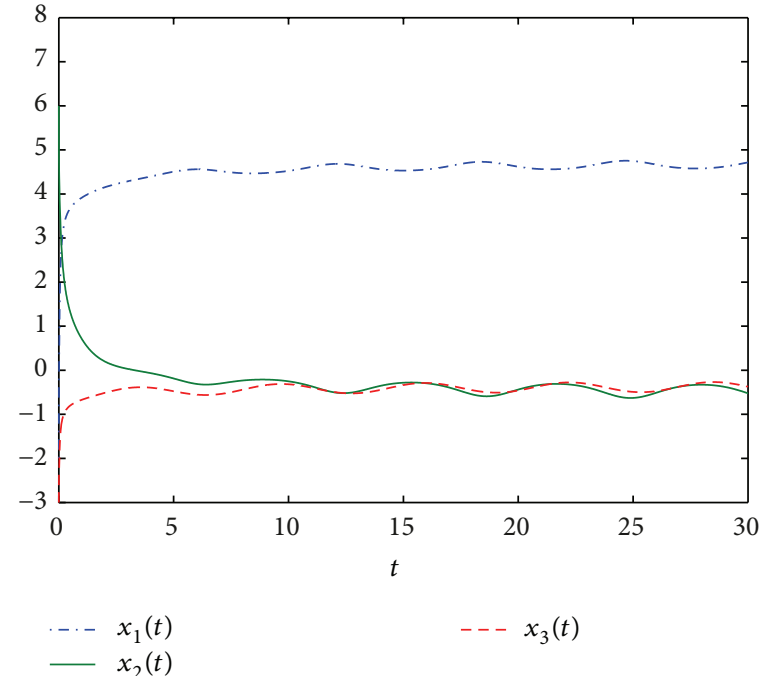

(b)

Figure 2: The states of system (52) with $A=\operatorname{diag}\{5,4,4\}:$ (a) $\alpha=0.9$, (b) $\alpha=0.6$.

\section{Numerical Simulations}

The effectiveness of the obtained theoretical results is demonstrated by the following examples. The predictor-corrector scheme is used for the approximate numerical solutions of the fractional-order neural networks.

5.1. An Example of Robust Stability for Fractional-Order Hopfield Neural Networks with Parameter Uncertainties. For system (18), consider the following neural networks of $n=3$ neurons with hub structure [26]. Let $f(x)=$ $\left(\sin \left(x_{1}\right), \tanh \left(x_{2}\right), \tanh \left(x_{3}\right)\right)^{T}, w=(8 \pi, 0,0)^{T}, \Delta A(t)=$ $\operatorname{diag}\{0,0.6 \sin (t), 0.4 \cos (t)\}$, and

$$
\begin{gathered}
B=\left[\begin{array}{ccc}
3 & -2 & -2 \\
1 & 1 & 0 \\
1 & 0 & 1
\end{array}\right], \\
\Delta B(t)=\left[\begin{array}{ccc}
0 & 0 & 0.8 \sin (t) \\
0.5 \arctan (t) & 1.2 \cos (t) & 0 \\
0.4 \cos (t) & 0 & 0.5 \cos (t)
\end{array}\right] .
\end{gathered}
$$

Then, system (18) can be written as

$$
\begin{aligned}
{ }_{0} D_{t}^{\alpha} x_{1}= & -a_{11} x_{1}+3 \sin \left(x_{1}\right)-2 \tanh \left(x_{2}\right) \\
& -(2-0.8 \sin (t)) \tanh \left(x_{3}\right)+8 \pi, \\
{ }_{0} D_{t}^{\alpha} x_{2}= & -\left(a_{22}+0.6 \sin (t)\right) x_{2} \\
& +(1+0.5 \arctan (t)) \sin \left(x_{1}\right) \\
& +(1+1.2 \cos (t)) \tanh \left(x_{2}\right), \\
{ }_{0} D_{t}^{\alpha} x_{3}= & -\left(a_{33}+0.4 \cos (t)\right) x_{3} \\
& +(1+0.4 \cos (t)) \sin \left(x_{1}\right) \\
& +(1+0.5 \cos (t)) \tanh \left(x_{3}\right) .
\end{aligned}
$$

Obviously, we know that $L=\operatorname{diag}\{1,1,1\}$ and $\|L\|=1$ in system (52). When $a_{11}=5$ and $a_{22}=a_{33}=4$, Figure 2 is the solution of system (52) with different $\alpha$ and initial value $(-2,6,-3)^{T}$. Then, we choose $a_{11}=a_{22}=a_{33}=7, \beta=$ $\operatorname{diag}\{2,1,1\}$, and $\|\beta\|=2$, so $\left(A_{1}\right)-\left(A_{3}\right)$ hold with the existing $\lambda=0.2$. According to Theorem 14 , the unique equilibrium point $\bar{x}=(\pi, 0,0)^{T}$ of system (52) is globally robust stable. With same $\alpha$ and initial value in Figure 2, Figure 3 shows that the solution of system (52) converges to the equilibrium point $\bar{x}=(\pi, 0,0)^{T}$ which verifies the effectiveness of Theorem 14 .

5.2. An Example of Synchronization for Fractional-Order Hopfield Neural Networks with Parameter Uncertainties. In systems (40) and (41), consider the following neural networks of $n=3$ neurons [37]. Let $f(x)=\left(\tanh \left(x_{1}\right), \tanh \left(x_{2}\right)\right.$, $\left.\tanh \left(x_{3}\right)\right)^{T}, w=(0,0,0)^{T}, A=\operatorname{diag}\{1,1,1\}$, and

$$
B=\left[\begin{array}{ccc}
2 & -1.2 & 0 \\
1.8 & 1.71 & 1.15 \\
-4.75 & 0 & 1.1
\end{array}\right]
$$

Case 1. When $\Delta A(t)=\Delta C(t)=\operatorname{diag}\{0.5 \sin (t), 0,0.2 \cos (t)\}$ and

$$
\Delta B(t)=\Delta D(t)=\left[\begin{array}{ccc}
0 & 0.2 \cos (t) & 0 \\
0.1 \sin (t) & 0 & 0 \\
0 & 0 & 0.3 \cos (t)
\end{array}\right]
$$

systems (40) and (41) have same parameter uncertainties. So the drive-resonse systems can be written as

$$
\begin{aligned}
{ }_{0} D_{t}^{\alpha} x_{1}= & -(1+0.5 \sin (t)) x_{1}+2 \tanh \left(x_{1}\right) \\
& -(1.2-0.2 \cos (t)) \tanh \left(x_{2}\right)
\end{aligned}
$$




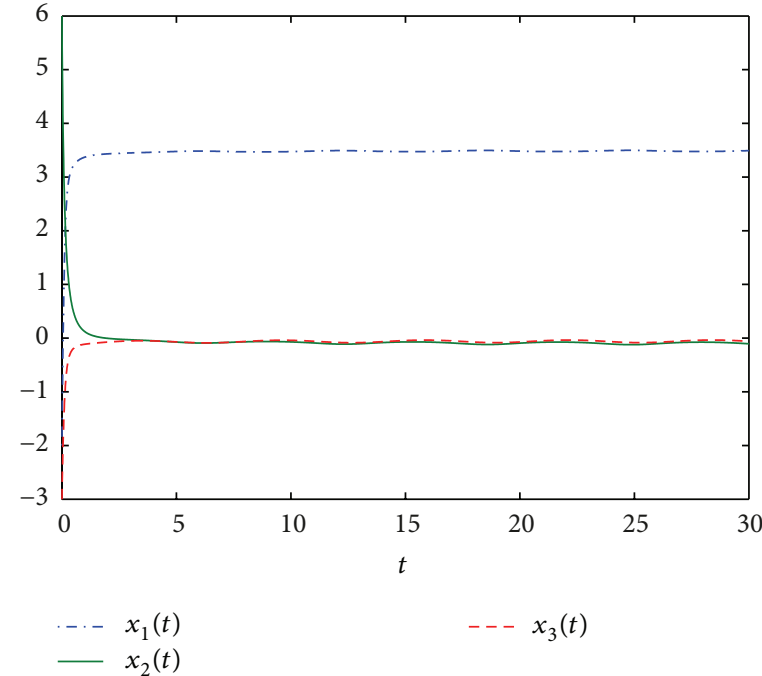

(a)

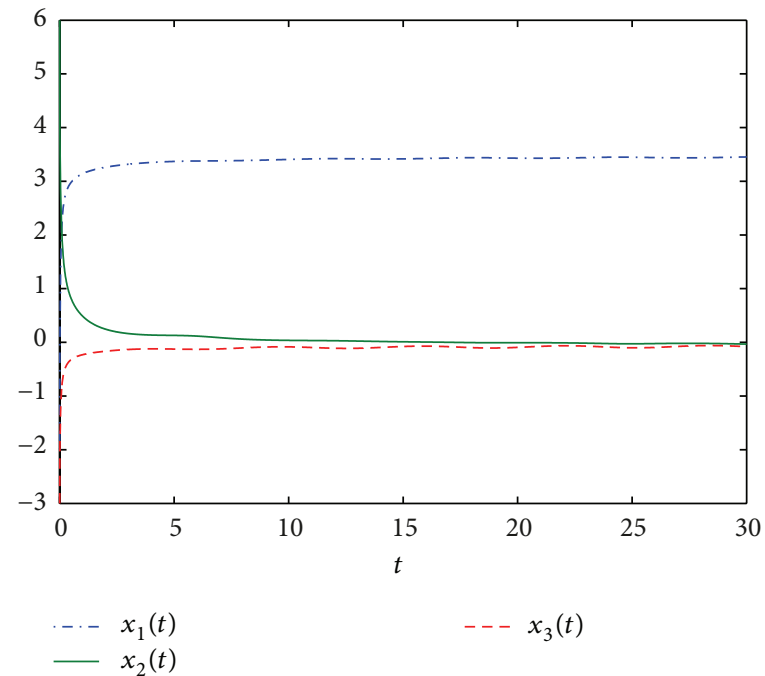

(b)

Figure 3: The states of system (52) with $A=\operatorname{diag}\{7,7,7\}:$ (a) $\alpha=0.9$, (b) $\alpha=0.6$.

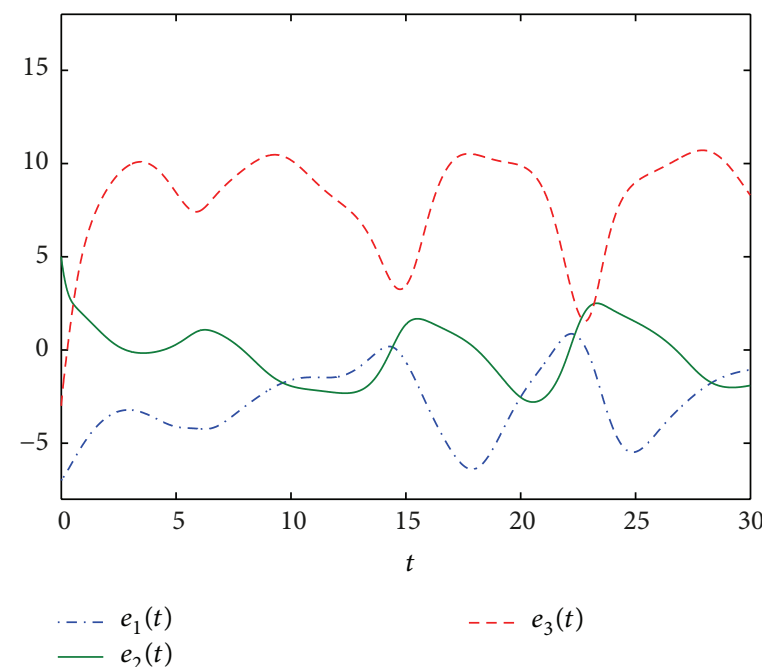

(a)

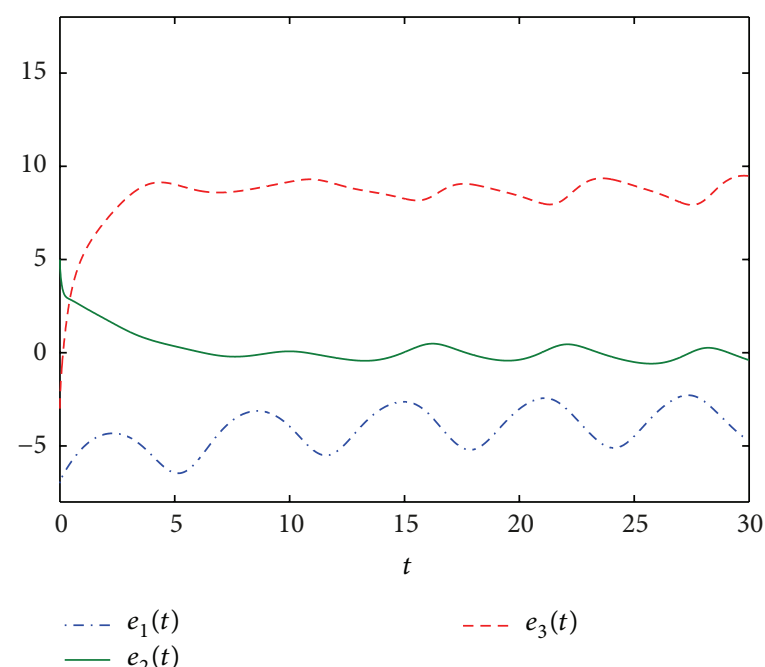

(b)

FIgURE 4: The state error graph of drive-response systems (55) and (56) without control: (a) $\alpha=0.9$, (b) $\alpha=0.6$.

$$
\begin{aligned}
{ }_{0} D_{t}^{\alpha} x_{2}= & -x_{2}+(1.8+0.1 \sin (t)) \tanh \left(x_{1}\right) \\
& +1.71 \tanh \left(x_{2}\right)+1.15 \tanh \left(x_{3}\right), \\
{ }_{0} D_{t}^{\alpha} x_{3}= & -(1+0.2 \cos (t)) x_{3}-4.75 \tanh \left(x_{1}\right) \\
& +(1.1+0.3 \cos (t)) \tanh \left(x_{3}\right), \\
{ }_{0} D_{t}^{\alpha} y_{1}= & -(1+0.5 \sin (t)) y_{1}+2 \tanh \left(y_{1}\right) \\
& -(1.2-0.2 \cos (t)) \tanh \left(y_{2}\right)-k e_{1}, \\
{ }_{0} D_{t}^{\alpha} y_{2}= & -y_{2}+(1.8+0.1 \sin (t)) \tanh \left(y_{1}\right) \\
& +1.71 \tanh \left(y_{2}\right)+1.15 \tanh \left(y_{3}\right)-k e_{2},
\end{aligned}
$$

If the respond system (56) is not controlled by control law, that is, $u(t)=0$, the error states are shown in Figure 4 with $(-4,1,-1)^{T} . L=\operatorname{diag}\{1,1,1\}$ and $\|L\|=1$ are obtained in systems (55) and (56). $\beta=\operatorname{diag}\{3,1,1\},\|\beta\|=3$, and the control parameter $k=5$ are chosen, such that $\left(A_{1}\right),\left(A_{2}\right)$, and (37) are satisfied with the existing $\lambda=0.69$. According to Theorem 17, the robust synchronization between systems (55) and (56) can be realized. With same $\alpha$ and initial values in Figure 4, the error states are shown with converging to 0 in Figure 5. 


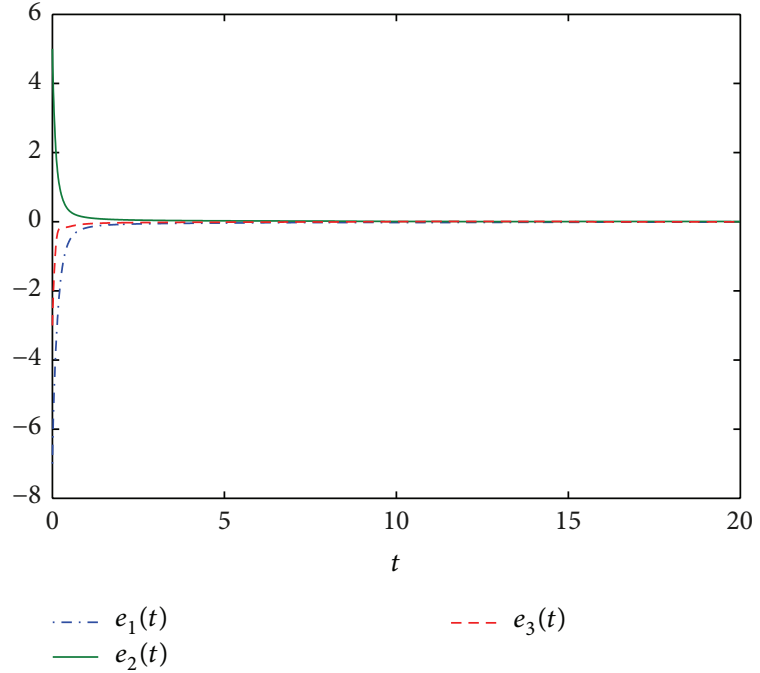

(a)

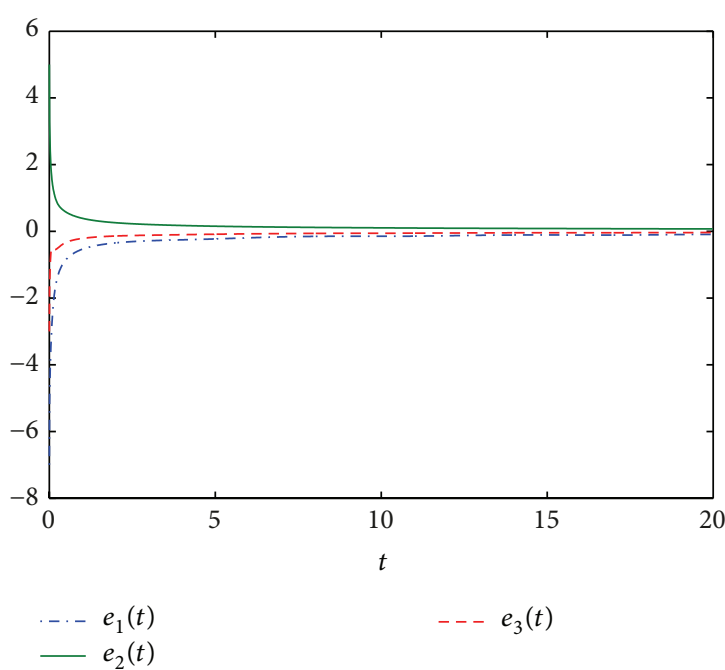

(b)

FIgURE 5: The state error graph of drive-response systems (55) and (56) under control: (a) $\alpha=0.9$, (b) $\alpha=0.6$.

Remark 22. In [37], authors pointed out that convergent rate is getting bigger with the increasing of fractional-order $\alpha$. From Figures 3 and 5 , the convergent speed with $\alpha=0.9$ is faster than the one with $\alpha=0.6$, which coincides with their results.

Case 2. If $\Delta A(t)=\Delta B(t)=0.1 e^{-t} I, \Delta C(t)=\operatorname{diag}\{0.1 \sin (t)$, $0,0.1 \cos (t)\}$, and

$$
\Delta D(t)=\left[\begin{array}{ccc}
0 & 0.9 \cos (t) & 0 \\
0.8 \sin (t) & 0 & 0 \\
0 & 0 & \cos (t)
\end{array}\right],
$$

systems (40) and (41) have different parameter uncertainties. Then, the drive-response systems can be described as

$$
\begin{aligned}
{ }_{0} D_{t}^{\alpha} x_{1}= & -\left(1+0.1 e^{-t}\right) x_{1} \\
& +\left(2+0.1 e^{-t}\right) \tanh \left(x_{1}\right)-1.2 \tanh \left(x_{2}\right), \\
{ }_{0} D_{t}^{\alpha} x_{2}= & -\left(1+0.1 e^{-t}\right) x_{2}+1.8 \tanh \left(x_{1}\right) \\
& +\left(1.71+0.1 e^{-t}\right) \tanh \left(x_{2}\right)+1.15 \tanh \left(x_{3}\right), \\
{ }_{0} D_{t}^{\alpha} x_{3}= & -\left(1+0.1 e^{-t}\right) x_{3}-4.75 \tanh \left(x_{1}\right) \\
& +\left(1.1+0.1 e^{-t}\right) \tanh \left(x_{3}\right),
\end{aligned}
$$$$
{ }_{0} D_{t}^{\alpha} y_{1}=-(1+0.1 \sin (t)) y_{1}+2 \tanh \left(y_{1}\right)
$$$$
-(1.2-0.9 \cos (t)) \tanh \left(y_{2}\right)-k e_{1},
$$$$
{ }_{0} D_{t}^{\alpha} y_{2}=-y_{2}+(1.8+0.8 \sin (t)) \tanh \left(y_{1}\right)
$$$$
+1.71 \tanh \left(y_{2}\right)+1.15 \tanh \left(y_{3}\right)-k e_{2},
$$

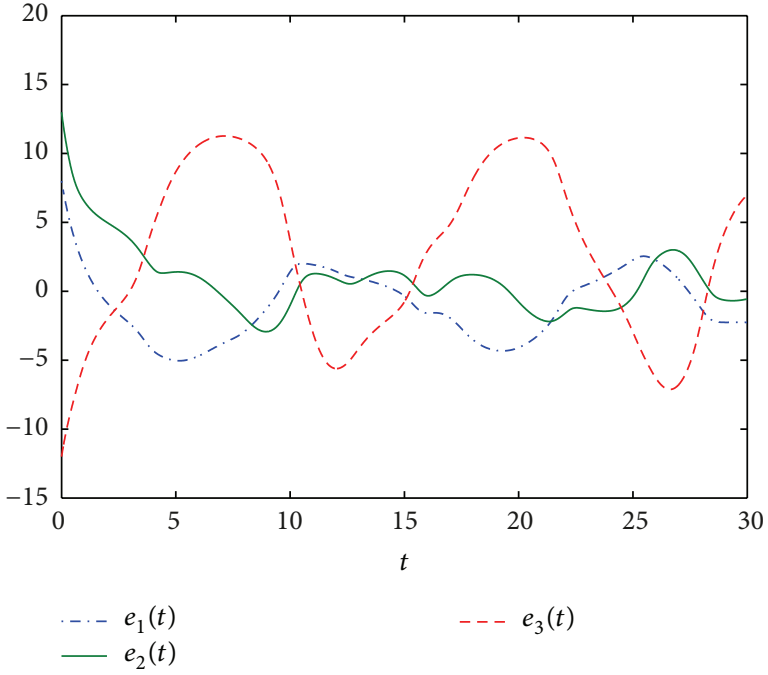

FIGURE 6: The state error graph of drive-response systems (58) and (59) without control.

$$
\begin{aligned}
{ }_{0} D_{t}^{\alpha} y_{3}= & -(1+0.1 \cos (t)) y_{3}-4.75 \tanh \left(y_{1}\right) \\
& +(1.1+\cos (t)) \tanh \left(y_{3}\right)-k e_{3} .
\end{aligned}
$$

When the respond system (59) is not controlled by control law, that is, $u(t)=0$, the error states are shown in Figure 6 with $\alpha=0.98$ and initial values $x(0)=(2,-5,3)^{T}$ and $y(0)=(10,8,-9)^{T}$. Similarly, $L=\operatorname{diag}\{1,1,1\}$ and $\|L\|=1$ are gained in systems (58) and (59). With $\alpha=0.98$, the chaotic behaviors of drive system (58) are shown in Figure 7, which have been studied in [37]. And $\delta=10, \delta_{f}=1$, and $\theta=6.3 \mathrm{can}$ be obtained from the state graph of system (58). If the quasisynchronization between systems (55) and (56) is wanted to 


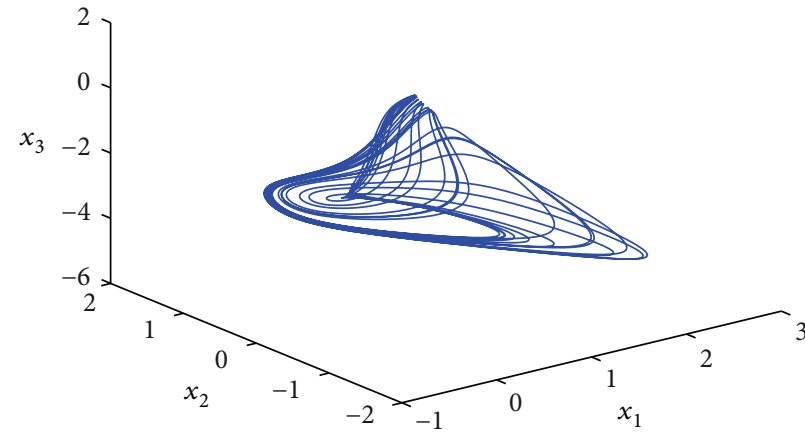

(a)

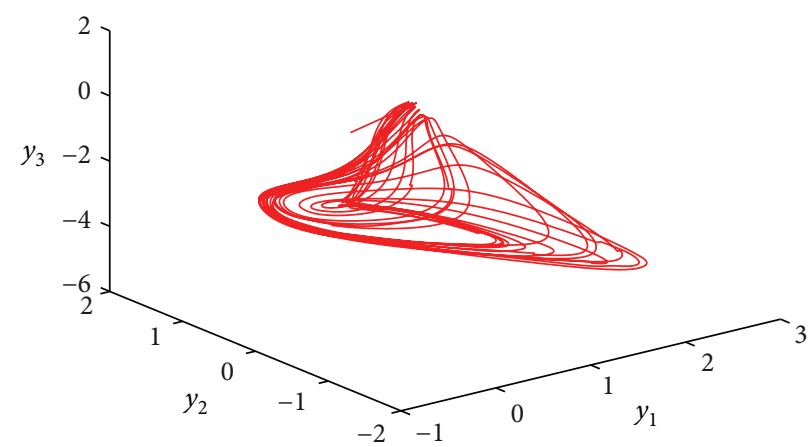

(b)

Figure 7: (a) The states of drive system (58) with chaotic behaviors. (b) The states of response system (59) with chaotic behaviors under control.

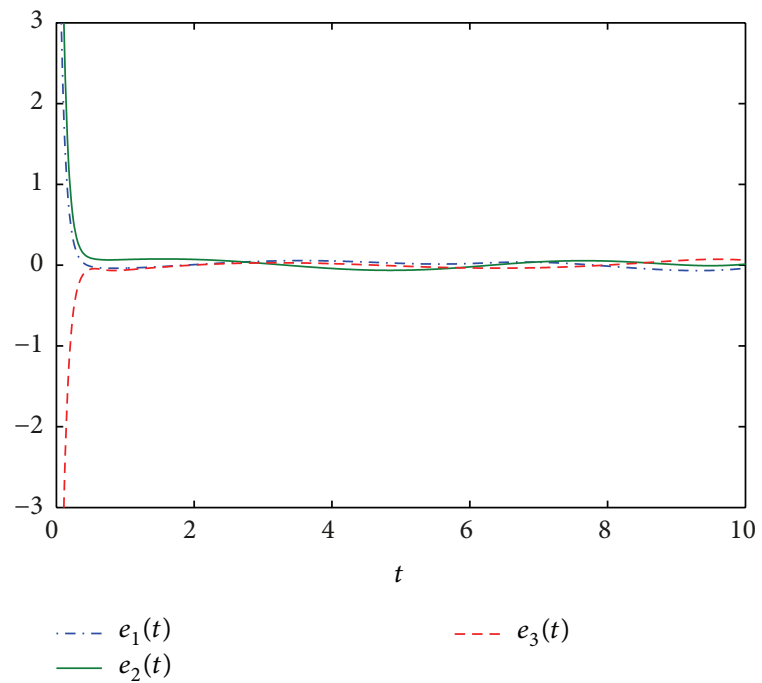

(a)

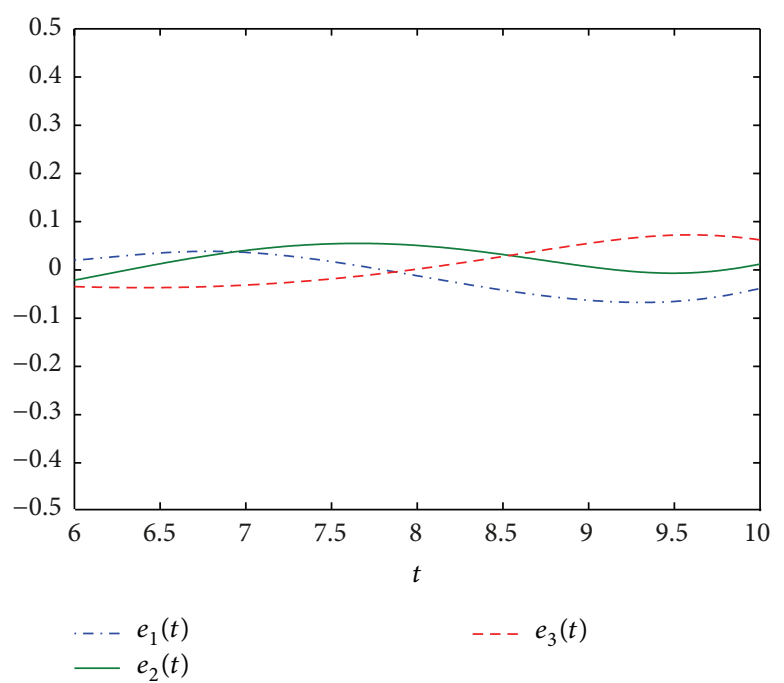

(b)

Figure 8: (a) The state error graph of drive-response systems (58) and (59) under control. (b) The state error zoom graph from time 6 to 10.

be realized with error bound $\varphi=2$, the control parameter $k=11.85$ should be chosen based on Theorem 20, such that $\left(A_{2}\right)$ and $\left(A_{4}\right)-\left(A_{6}\right)$ are satisfied with the existing $\lambda=3.2$. With same $\alpha$ and initial values in Figure 6, the error states are shown in Figure 8 and $\left|e_{i}(t)\right| \leq\|e(t)\| \leq 2$ for $i=1,2,3$ is gained as $t>1$.

\section{Conclusion}

By employing appropriate Lyapunov functionals, robust stability and synchronization for fractional-order Hopfield neural networks with parameter uncertainties are studied. Based on the fractional-order Lyapunov direct method, the sufficient condition of the existence, uniqueness, and globally robust stability of the equilibrium point is presented. Moreover, the sufficient condition of the robust synchronization between such neural systems with same parameter uncertainties is proposed owing to the robust stability analysis of its synchronization error system. In addition, for different parameter uncertainties, the quasi-synchronization between the class of neural networks is investigated with linear control. And the quasi-synchronization error bound can be controlled as we want by choosing suitable control parameters. The effectiveness of theoretical results is verified by the numerical simulations.

For the fractional-order delayed neural networks (FDNN), we try to discuss the robust stability and synchronization of FDNN with parameter uncertainties. Furthermore, the issue of robust stability for such system with stochastic external inputs is very novel and interesting, which is an important topic in our next work.

\section{Conflict of Interests}

The authors declare that there is no conflict of interests regarding the publication of this paper.

\section{Acknowledgment}

This work is supported by the National Nature Science Foundation of China (no. 11371049). 


\section{References}

[1] H. Zhang and Y. Xia, "Existence and exponential stability of almost periodic solution for Hopfield-type neural networks with impulse," Chaos, Solitons and Fractals, vol. 37, no. 4, pp. 1076-1082, 2008.

[2] H. Xiang, K.-M. Yan, and B.-Y. Wang, "Existence and global exponential stability of periodic solution for delayed high-order Hopfield-type neural networks," Physics Letters A: General, Atomic and Solid State Physics, vol. 352, no. 4-5, pp. 341-349, 2006.

[3] Y. Wang, C. Lu, G. Ji, and L. Wang, "Global exponential stability of high-order Hopfield-type neural networks with S-type distributed time delays," Communications in Nonlinear Science and Numerical Simulation, vol. 16, no. 8, pp. 3319-3325, 2011.

[4] X.-Y. Lou and B.-T. Cui, "Novel global stability criteria for highorder Hopfield-type neural networks with time-varying delays," Journal of Mathematical Analysis and Applications, vol. 330, no. 1, pp. 144-158, 2007.

[5] J. Cao and T. Chen, "Globally exponentially robust stability and periodicity of delayed neural networks," Chaos, Solitons and Fractals, vol. 22, no. 4, pp. 957-963, 2004.

[6] T. Ensari and S. Arik, "New results for robust stability of dynamical neural networks with discrete time delays," Expert Systems with Applications, vol. 37, no. 8, pp. 5925-5930, 2010.

[7] O. Faydasicok and S. Arik, "Further analysis of global robust stability of neural networks with multiple time delays," Journal of the Franklin Institute, vol. 349, no. 3, pp. 813-825, 2012.

[8] O. Faydasicok and S. Arik, "Robust stability analysis of a class of neural networks with discrete time delays," Neural Networks, vol. 29-30, pp. 52-59, 2012.

[9] $\mathrm{H}$. Yu and H. Wu, "Global robust exponential stability for Hopfield neural networks with non-Lipschitz activation functions," Journal of Mathematical Sciences, vol. 187, no. 4, pp. 511-523, 2012.

[10] S. Xu, J. Lam, and D. W. C. Ho, "Novel global robust stability criteria for interval neural networks with multiple time-varying delays," Physics Letters A: General, Atomic and Solid State Physics, vol. 342, no. 4, pp. 322-330, 2005.

[11] P. Balasubramaniam, V. Vembarasan, and R. Rakkiyappan, "Delay-dependent robust asymptotic state estimation of TakagiSugeno fuzzy Hopfield neural networks with mixed interval time-varying delays," Expert Systems with Applications, vol. 39, no. 1, pp. 472-481, 2012.

[12] P. Balasubramaniam, V. Vembarasan, and R. Rakkiyappan, "Global robust asymptotic stability analysis of uncertain switched Hopfield neural networks with time delay in the leakage term," Neural Computing and Applications, vol. 21, no. 7, pp. 1593-1616, 2012.

[13] H. Lu, "Chaotic attractors in delayed neural networks," Physics Letters A: General, Atomic and Solid State Physics, vol. 298, no. 2-3, pp. 109-116, 2002.

[14] Y. Shen and J. Wang, "An improved algebraic criterion for global exponential stability of recurrent neural networks with timevarying delays," IEEE Transactions on Neural Networks, vol. 19, no. 3, pp. 528-531, 2008.

[15] M. Chen and W.-H. Chen, "Robust adaptive neural network synchronization controller design for a class of time delay uncertain chaotic systems," Chaos, Solitons and Fractals, vol. 41, no. 5, pp. 2716-2724, 2009.

[16] P. Li, J. Cao, and Z. Wang, "Robust impulsive synchronization of coupled delayed neural networks with uncertainties," Physica A:
Statistical Mechanics and its Applications, vol. 373, pp. 261-272, 2007.

[17] L. Pan and J. Cao, "Stochastic quasi-synchronization for delayed dynamical networks via intermittent control," Communications in Nonlinear Science and Numerical Simulation, vol. 17, no. 3, pp. 1332-1343, 2012.

[18] X. Liu, J. Cao, and W. Yu, "Filippov systems and quasi-synchronization control for switched networks," Chaos, vol. 22, no. 3, Article ID 033110, 2012.

[19] W. Zhang, J. Huang, and P. Wei, "Weak synchronization of chaotic neural networks with parameter mismatch via periodically intermittent control," Applied Mathematical Modelling, vol. 35, no. 2, pp. 612-620, 2011.

[20] R. L. Bagley and R. A. Calico, "Fractional order state equations for the control of viscoelastically damped structures," Journal of Guidance, Control, and Dynamics, vol. 14, no. 2, pp. 304-311, 1991.

[21] T. T. Hartley and C. F. Lorenzo, "Dynamics and control of initialized fractional-order systems," Nonlinear Dynamics, vol. 29, no. 1-4, pp. 201-233, 2002.

[22] G. Cottone, M. D. Paola, and R. Santoro, "A novel exact representation of stationary colored Gaussian processes (fractional differential approach)," Journal of Physics A: Mathematical and Theoretical, vol. 43, no. 8, Article ID 085002, 2010.

[23] E. Reyes-Melo, J. Martinez-Vega, C. Guerrero-Salazar, and U. Ortiz-Mendez, "Application of fractional calculus to the modeling of dielectric relaxation phenomena in polymeric materials," Journal of Applied Polymer Science, vol. 98, no. 2, pp. 923-935, 2005.

[24] A. Boroomand and M. B. Menhaj, "Fractional-order hopfield neural networks," in Advances in Neuro-Information Processing, vol. 5506, pp. 883-890, Springer, Berlin, Germany, 2009.

[25] B. N. Lundstrom, M. H. Higgs, W. J. Spain, and A. L. Fairhall, "Fractional differentiation by neocortical pyramidal neurons," Nature Neuroscience, vol. 11, no. 11, pp. 1335-1342, 2008.

[26] E. Kaslik and S. Sivasundaram, "Nonlinear dynamics and chaos in fractional-order neural networks," Neural Networks, vol. 32, pp. 245-256, 2012.

[27] J. Sabatier, M. Moze, and C. Farges, "LMI stability conditions for fractional order systems," Computers and Mathematics with Applications, vol. 59, no. 5, pp. 1594-1609, 2010.

[28] Z. Liao, C. Peng, W. Li, and Y. Wang, "Robust stability analysis for a class of fractional order systems with uncertain parameters," Journal of the Franklin Institute, vol. 348, no. 6, pp. 11011113, 2011.

[29] Y. D. Ma, J. G. Lu, W. D. Chen et al., "Robust stability bounds of uncertain fractional-order systems," Fractional Calculus and Applied Analysis, vol. 17, no. 1, pp. 136-153, 2014.

[30] S. Kuntanapreeda, "Robust synchronization of fractional-order unified chaotic systems via linear control," Computers and Mathematics with Applications, vol. 63, no. 1, pp. 183-190, 2012.

[31] W. K. Wong, H. Li, and S. Y. S. Leung, "Robust synchronization of fractional-order complex dynamical networks with parametric uncertainties," Communications in Nonlinear Science and Numerical Simulation, vol. 17, no. 12, pp. 4877-4890, 2012.

[32] Y. Li, Y. Chen, and I. Podlubny, "Stability of fractional-order nonlinear dynamic systems: lyapunov direct method and generalized Mittag-Leffler stability," Computers and Mathematics with Applications, vol. 59, no. 5, pp. 1810-1821, 2010.

[33] Z. Zhang and H. Jiang, "Quasi-synchronization of different fractional-order chaotic systems with external perturbations 
and its application," in Advances in Neural Networks-ISNN 2012, pp. 256-265, Springer, Berlin, Germany, 2012.

[34] I. Podlubny, Fractional Differential Equations, Academic Press, London, UK, 1999.

[35] A. A. Kilbas, H. M. Srivastava, and J. J. Trujillo, Theory and Application of Fractional Differential Equations, Elsevier, New York, NY, USA, 2006.

[36] H. Delavari, D. Baleanu, and J. Sadati, "Stability analysis of Caputo fractional-order nonlinear systems revisited," Nonlinear Dynamics, vol. 67, no. 4, pp. 2433-2439, 2012.

[37] J. Yu, C. Hu, and H. Jiang, " $\alpha$-stability and $\alpha$-synchronization for fractional-order neural networks," Neural Networks, vol. 35, pp. 82-87, 2012. 


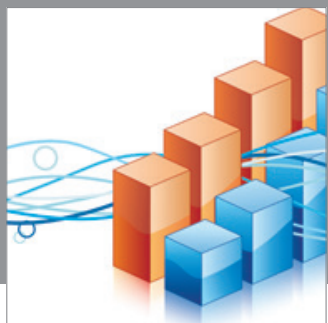

Advances in

Operations Research

mansans

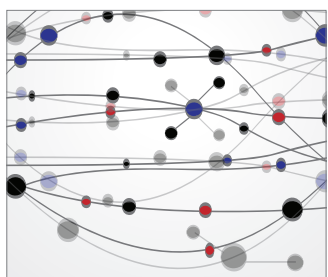

The Scientific World Journal
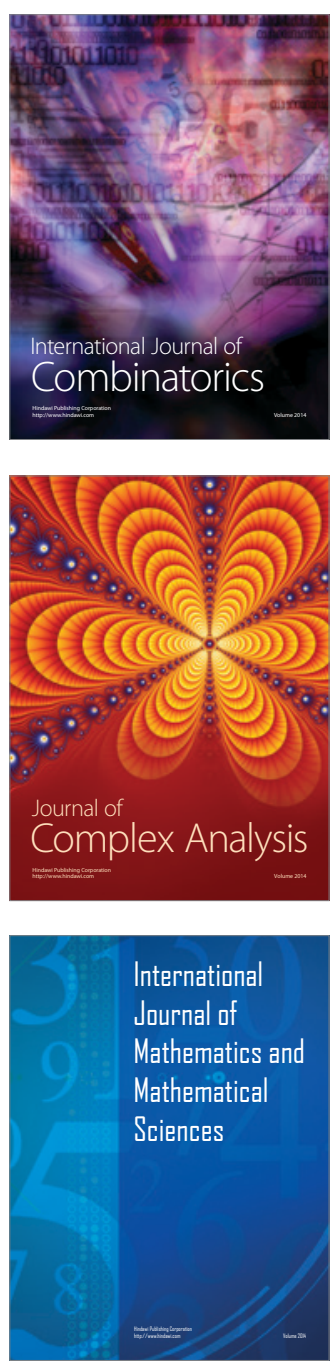
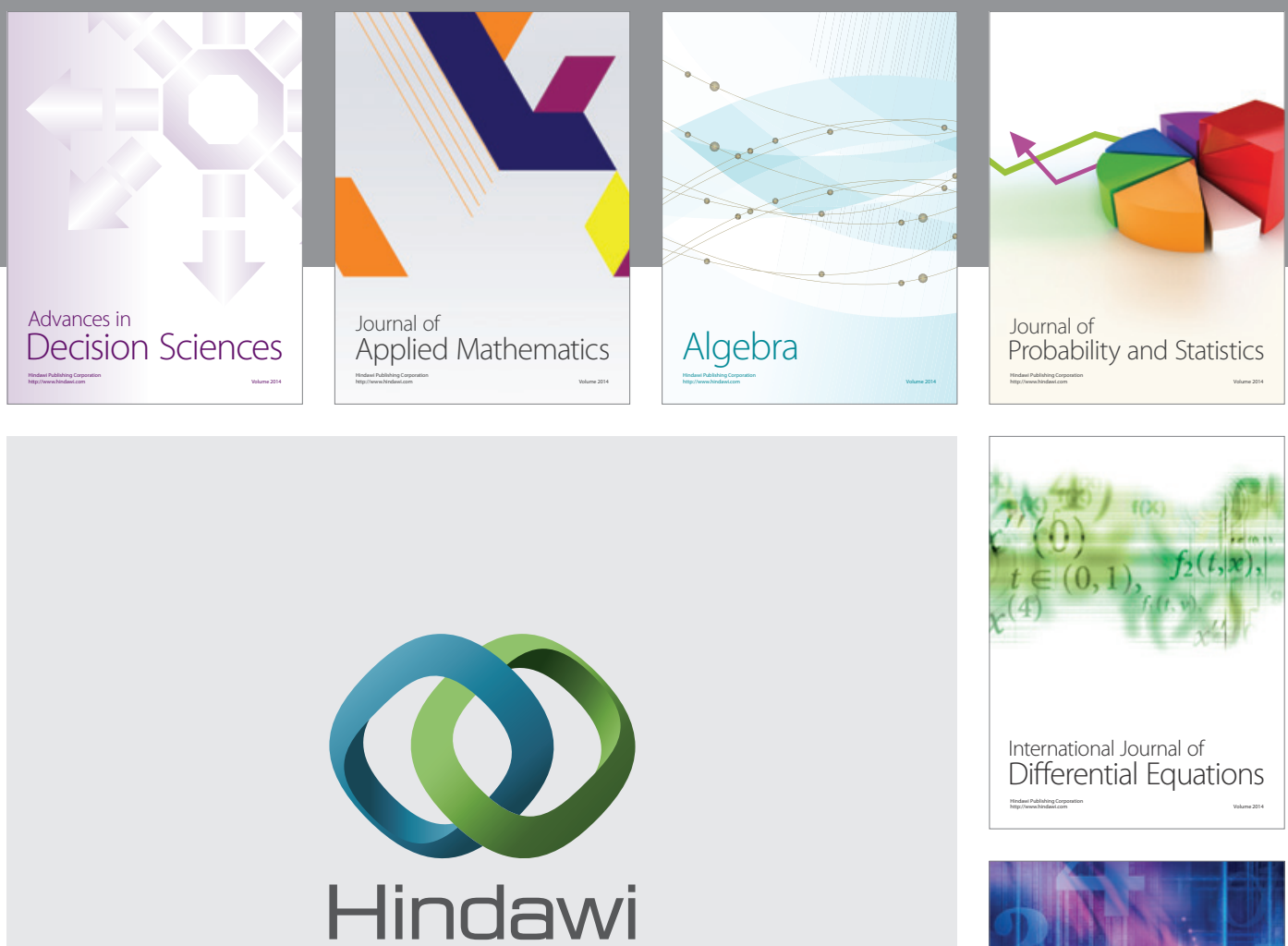

Submit your manuscripts at http://www.hindawi.com
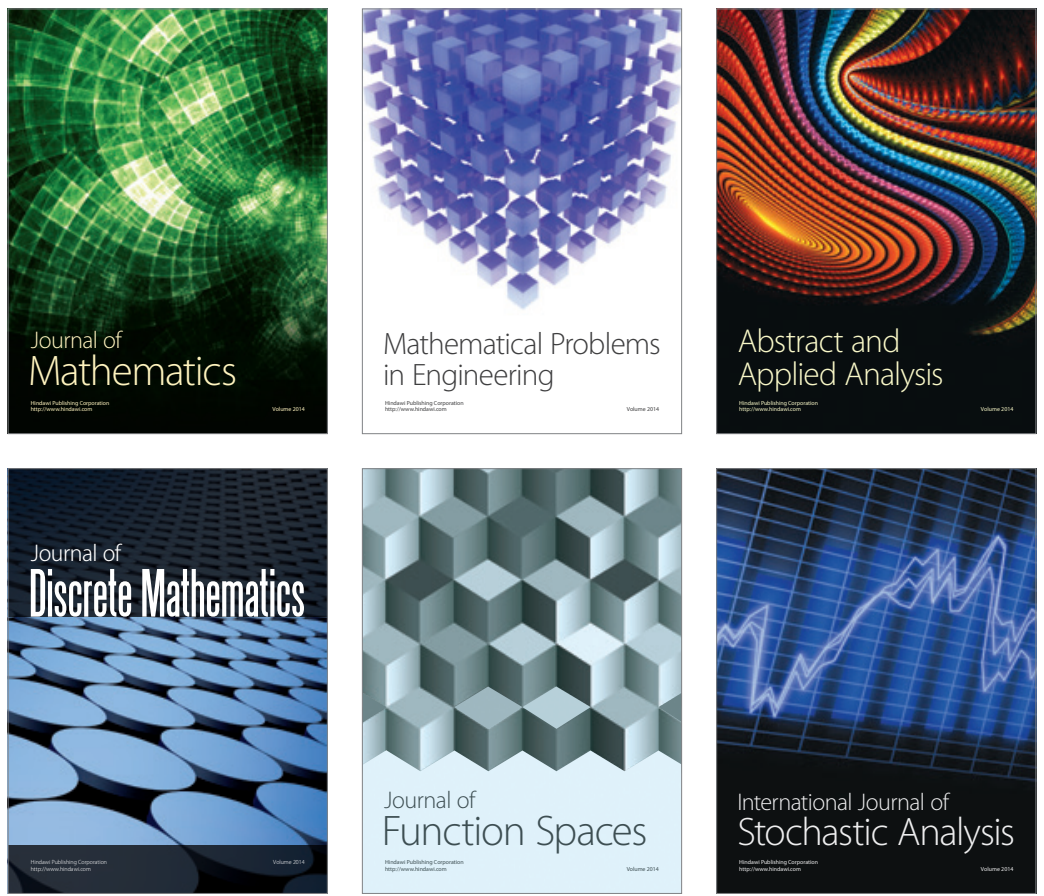

Journal of

Function Spaces

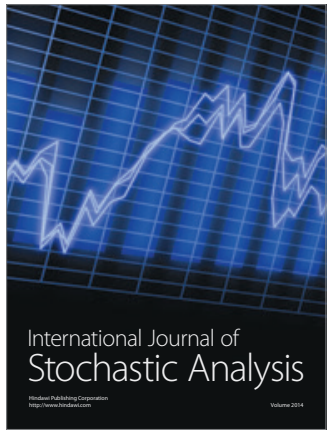

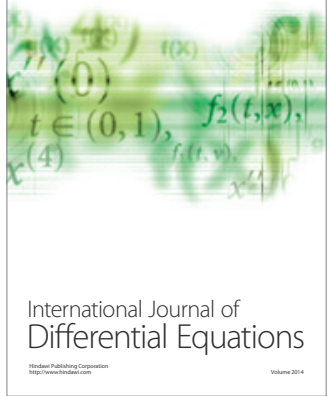
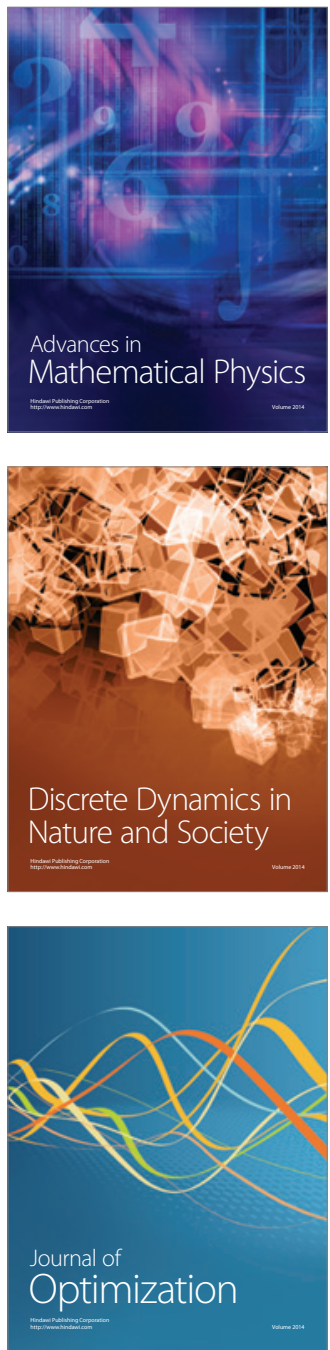